\title{
Dynamical Characteristics of External Cavity Quantum Cascade Lasers
}

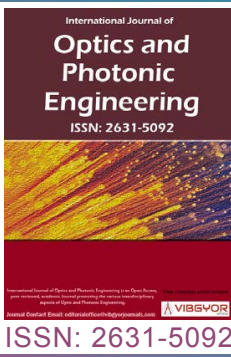

\section{Abdelouahab Hamadou ${ }^{1,2^{*}}$}

${ }^{1}$ Département de Génie Civil, Faculté des Sciences et de la Technologie, Université Mohamed El Bachir El Ibrahimi, Bordj Bou Arreridj 34000, Algeria

${ }^{2}$ Laboratoire d'étude des Surfaces et Interfaces des Matériaux Solides (LESIMS), Université Ferhat Abbas Sétif1, 19000, Algeria

\begin{abstract}
In this paper, we calculate the dynamical characteristics such as delay time, rise time, and time to steady state establishment, of a mid infrared quantum cascade laser coupled to external cavity. The approach is based on the three-level rate equations including the dependence of the loss on external cavity parameters. We find in particular that the threshold current of external cavity is strongly influenced by the external reflectivity and external cavity length. In addition, the equations that allow for the determination of the dynamical characteristics are derived within the premises of our model in the general case. The effects of the external cavity parameters on dynamical characteristics are also explored.
\end{abstract}

\section{Keywords}

Quantum cascade laser, External cavity, Delay time, Rise time, Time to steady state establishment

\section{Introduction}

After the first demonstration of the quantum cascade (QC) laser by Faist in 1994 [1], external cavity (EC) QC laser was demonstrated for the first time by Luo in 2001 [2]. In recent years, different works on EC-QC lasers were published because of their useful characteristics such as spectral tuning rang and single mode operation [3-14].

It is well known that EC strongly affects the losses, photon lifetime and threshold current of $\mathrm{QC}$ laser, and thus influence the turn on delay time where it decreases with increasing external reflectivity $[10,11,14,15]$. Equally as important for the EC-QC laser operation is the delay time, rise time, and time to steady state establishment (TSSE). Therefore, studies of EC-QC laser are important for potential applications in intersubband photonics field. In this paper, we calculate the delay time $\left(t_{d}^{E C}\right)$, the rise time ( $\left.\Delta t_{\text {rise }}^{E C}\right)$, and the TSSE $\left(t_{s s}^{E C}\right)$ in terms of current injection, threshold current, scattering times, and photon lifetime for the external cavity, using a rate equations model. It is an extension of the

*Corresponding author: Abdelouahab Hamadou, Département de Génie Civil, Faculté des Sciences et de la Technologie, Université Mohamed El Bachir El Ibrahimi, Bordj Bou Arreridj 34000, Algeria; Laboratoire d'étude des Surfaces et Interfaces des Matériaux Solides (LESIMS), Université Ferhat Abbas Sétif1 19000, Algeria, Tel: +213776937575

Accepted: December 10, 2019; Published: December 12, 2019

Copyright: @ 2019 Hamadou A. This is an open-access article distributed under the terms of the Creative Commons Attribution License, which permits unrestricted use, distribution, and reproduction in any medium, provided the original author and source are credited.

Hamadou. Int J Opt Photonic Eng 2019, 4:020 
previous work reported in Ref [15].

\section{The Model}

Figure 1 shows the schematic illustration of a cavity model for the QC laser with external cavity that we treat here. The output power from a Fabry-Perot QC laser cavity (length $L$ and reflectivities $\mathrm{R}_{1}$ and $R_{2}$ ) is reflected by an external mirror of reflectance $R_{\text {ext }}$, which is located at a distance $L_{\text {ext }}$ from the front facet of the QC laser. The dynamics of ECQC laser can be described by a three-level model. The upper and lower states will be taken as levels 3 and 2, respectively, while the ground state used to empty the lower state through longitudinal optical phonon (LO) emission will be called level 1.

The system of rate equations for electron numbers $N_{1}, N_{2}$ and $N_{3}$ in levels 1,2 and 3 , and the photon numbers $S^{F P}$ and $S^{E C}$ in the FP and in the EC is used to model the dynamic behavior of EC-QC laser in this work [15].

$$
\begin{aligned}
& \frac{d N_{3}}{d t}=\frac{I_{i n j}}{e}-\frac{N_{3}}{\tau_{3}}-\left(G^{F P} S^{F P}+G^{E C} S^{E C}\right)\left(N_{3}-N_{2}\right), \\
& \frac{d N_{2}}{d t}=\frac{N_{3}}{\tau_{32}}-\frac{N_{2}}{\tau_{21}}+\left(G^{F P} S^{F P}+G^{E C} S^{E C}\right)\left(N_{3}-N_{2}\right), \\
& \frac{d N_{1}}{d t}=\frac{N_{3}}{\tau_{31}}+\frac{N_{2}}{\tau_{21}}-\frac{N_{1}}{\tau_{\text {out }}}, \\
& \frac{d S^{F P}}{d t}=N_{p} G^{F P}\left(N_{3}-N_{2}\right) S^{F P}+N_{p} \beta \frac{N_{3}}{\tau_{s p}}-\frac{S^{F P}}{\tau_{p}^{F P}}, \\
& \frac{d S^{E C}}{d t}=\frac{1}{\rho_{c a v}}\left(N_{p} G^{E C}\left(N_{3}-N_{2}\right) S^{E C}+N_{p} \beta \frac{N_{3}}{\tau_{s p}}-\frac{S^{E C}}{\tau_{p}^{E C}}\right) .
\end{aligned}
$$

Where $I_{i n j}$ is the injected current, $e$ is the electron charge, $\tau_{32}, \tau_{31}$, and $\tau_{21}$ are the nonradiative scattering times between the corresponding levels due to LO-phonon emission, $\tau_{s p}$ is the radiative spontaneous relaxation time between levels 3 and $2, \tau_{3}$ is the lifetime of the upper level and defined as $\tau_{3}=1 /\left(1 / \tau_{32}+1 / \tau_{31}\right), \tau_{\text {out }}$ is the electron escape time between two adjacent stages [16], $\beta$ defines the fraction of the spontaneous emission light emitted in the lasing mode [17], $N_{p}$ is the number of stages, $\rho_{c a v}=1+L_{e x t} /\left(n_{\text {eff }} L\right)$ is the ratio of optical path lengths of the FP and the external cavity [18] where $n_{\text {eff }}$ is the effective refractive index of FP active region, and $G^{E C}$ and $G^{E C}$ are the gain coefficients per period in the FP and in the external cavity respectively. The latter is defined through [14].

$$
G^{E C}=G^{F P} \frac{\gamma_{32}^{2}}{\left(h v^{E C}-h v^{F P}\right)^{2}+\gamma_{32}^{2}},
$$

Where $2 \gamma_{32}$ stands for the full width at half maximum of the electroluminescence spectrum between levels 3 and $2, h$ is the Planck constant, and $v$ is the lasing frequency.

The parameters $\tau_{p}^{F P}$ and $\tau_{p}^{E C}$ in Eqs.(1d-e) are respectively the lifetimes of the photons in the FP active region and in the EC [15] and given as function of waveguide loss of the cavity $\alpha_{w}$ and the mirrors losses by

$$
\begin{aligned}
\tau_{p}^{F P} & =\frac{2 L}{c^{\prime}} \frac{1}{\left(2 L \alpha_{w}-\ln \left(R_{1} R_{2}\right)\right)}, \\
E C & =\frac{2}{\prime} \frac{1}{\left(2 L_{w} \ln \left(R R_{\text {eff }}\right)\right)},
\end{aligned}
$$

Where $R_{\text {eff }}$ is the effective reflectivity of the equivalent EC-QC laser and can be written as [19].

$$
R_{e f f}=\frac{R_{2}+R_{e x t}+2 \sqrt{R_{2} R_{e x t}} \cos (\omega \tau)}{1+R_{2} R_{e x t}+2 \sqrt{R_{2} R_{e x t}} \cos (\omega \tau)},
$$

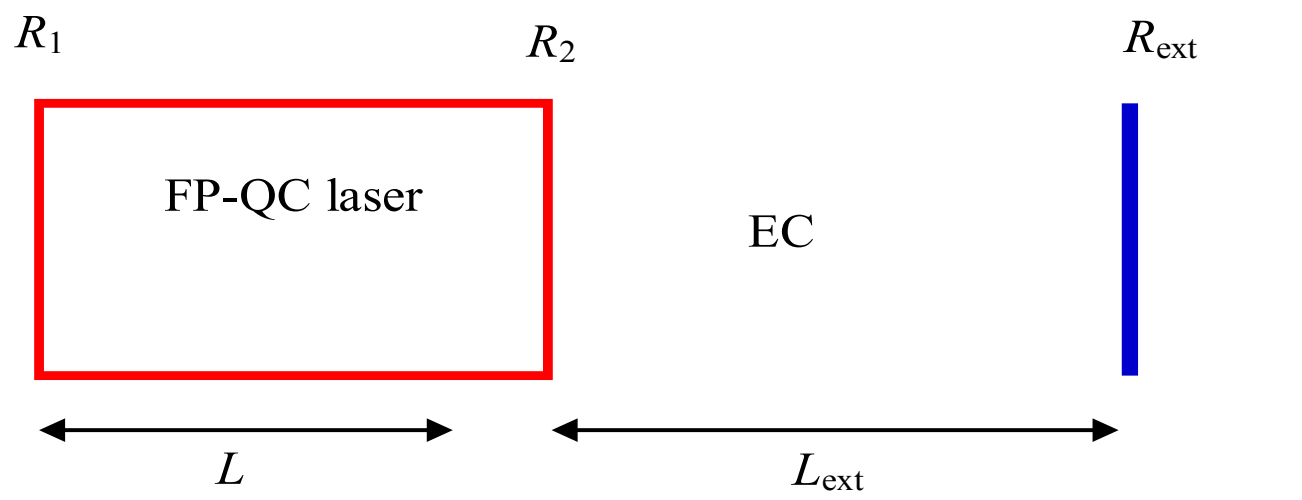

Figure 1: Schematic illustration of a cavity model for a QC laser with external cavity. 
Where $\omega$ is the laser angular frequency, $\tau=2 L_{e x t} / c$ is the round-trip time of light in the external cavity and where $c$ is the speed of light in vacuum.

In Eq. (3) we have assumed for simplicity that the waveguide loss of the EC mode is the same as that for the FP mode i.e., $\alpha_{w}^{E C}=\alpha_{w}^{F P}=\alpha_{w}$.

Under steady state conditions, the electron numbers in the upper and lower laser levels obey the following relations

$$
N_{3}=\frac{\frac{I_{i n j}}{e} \tau_{3}\left(1+\frac{\tau_{21}}{\tau_{3}\left(1+\frac{\tau_{21}}{\tau_{31}}\right)}\left(\frac{S^{F P}}{S_{s a t}^{F P}}+\frac{S^{E C}}{S_{s a t}^{E C}}\right)\right)}{1+\frac{S^{F P}}{S_{s a t}^{F P}}+\frac{S^{E C}}{S_{s a t}^{E C}}},
$$

$$
N_{2}=\frac{\frac{I_{i n j}}{e} \tau_{3}\left(\frac{\tau_{21}}{\tau_{32}}+\frac{\tau_{21}}{\tau_{3}\left(1+\frac{\tau_{21}}{\tau_{31}}\right)}\left(\frac{S^{F P}}{S_{s a t}^{F P}}+\frac{S^{E C}}{S_{s a t}^{E C}}\right)\right.}{1+\frac{S^{F P}}{S_{s a t}^{F P}}+\frac{S^{E C}}{S_{s a t}^{E C}}},
$$

Where we introduced the photon saturation number for both modes $S_{s a t}^{i}$ given by

$$
S_{s a t}^{i}=\frac{1}{\tau_{3}\left(1+\frac{\tau_{21}}{\tau_{31}}\right) G^{i}},
$$

Where the superscript $i$ refer to the FP or EC mode.

The population inversion $\Delta N$ between the upper and lower levels as a function of the FP and EC photon numbers can be then written by using Eqs. $(5 a-b)$ as

$$
\Delta N=\frac{\frac{I_{i n j}}{e} \tau_{3}\left(1-\frac{\tau_{21}}{\tau_{32}}\right)}{1+\frac{S^{F P}}{S_{\text {sat }}^{F P}}+\frac{S^{E C}}{S_{\text {sat }}^{E C}}} .
$$

Combining Eqs.(5a), (6), and (7) with Eq.(1e), the following expression is then obtained for ${ }^{E C}$ :

$$
S^{E C} \approx\left(\frac{I_{i n j}}{I_{t h}^{E C}}-1-\frac{S^{F P}}{S_{s a t}^{F P}}+\frac{1}{\left(1+\frac{\tau_{21}}{\tau_{31}}\right)} \frac{\beta}{\tau_{s p}} \frac{\tau_{21}}{\eta_{r}} \frac{I_{i n j}}{I_{t h}^{E C}}\right) S_{s a t}^{E C} .
$$

Where $I_{t h}^{E C}$ is the threshold current under the effect of external cavity while the parameter $\eta_{r}=\left(1-\tau_{21} / \tau_{32}\right) /\left(1+\tau_{21} / \tau_{31}\right)$ is the radiative efficiency.

At threshold condition, the rate of photon production inside the external cavity is equal to the rate of photon loss, i.e., $N_{p} G^{E C} \Delta N_{t h}^{E C}=1 / \tau_{p}^{E C}$, the threshold population inversion is given by

$$
\Delta N_{t h}^{E C}=\frac{1}{N_{p} \tau_{p}^{E C} G^{E C}} .
$$

The threshold current $I_{t h}^{E C}$ is obtained from Eq.(7) by setting $S^{F P}$ and $S^{E C}$ to zero and making $I_{t h}^{E C}$ replace $I_{i n j}$. With the help of Eq.(9) we get after easy algebra for $I_{t h}^{E C}$ the following expression

$$
I_{\text {th }}^{E C}=\frac{e}{N_{p} G^{E C} \tau_{3}\left(1-\frac{\tau_{21}}{\tau_{32}}\right) \tau_{p}^{E C}} .
$$

In the some way, the threshold current for the FP can be defined as

$$
I_{t h}^{F P}=\frac{e}{N_{p} G^{F P} \tau_{3}\left(1-\frac{\tau_{21}}{\tau_{32}}\right) \tau_{p}^{F P}} .
$$

Next using the theory developed above we estimate numerically $I_{t h}^{F P}, \tau_{p}^{F P}, G^{E C}$, and $S_{s a t}^{E C}$ using the following experimental $Q C$ laser parameters as reported in Refs. [14-16]: $L=1.5 \mathrm{~mm}, R_{1}=0.8$, $R_{2}=0.01, \alpha_{\mathrm{w}}=14 \mathrm{~cm}^{-1}, N_{\mathrm{p}}=48, \beta=1 \times 10^{-3}, n_{\text {eff }}=$ $3.27, \tau_{32}=2.4 \mathrm{ps}, \tau_{31}=3 \mathrm{ps}, \tau_{21}=0.4 \mathrm{ps}, \tau_{\text {out }}=1 \mathrm{ps}$, $\tau_{\mathrm{sp}}=140 \mathrm{~ns}, \lambda=8 \mu \mathrm{m}, G^{F P}=2 \times 10^{3} \mathrm{~s}^{-1}, \omega^{F P} \approx \omega^{E C}$. Our results are as follows: $\tau_{p}^{F P}=3.6 \mathrm{ps}, I_{t h}^{F P}=0.4$ A, $G^{E C}=2 \times 10^{3}$, and $S_{\text {sat }}^{E C}=3.17 \times 10^{8}$.

Figure 2 shows the variation of the threshold current $I_{t h}^{E C}$ with external cavity length $L_{e x t}$ for an external cavity reflectivity $R_{e x t}=90 \%$. As seen from this figure, the threshold current varies periodically with the external cavity length. The period of undulation is about $4 \mathrm{~cm}$. The low threshold current occurs at the $L_{\text {ext }}$ of around 4, 8 or 12 $\mathrm{cm}$ that produced minimum threshold current of 198.7 mA. In Figure 3, the threshold current $I_{t h}^{E C}$ is plotted versus external cavity reflectivity $R_{\text {ext }}$ for an external cavity length $L_{\text {ext }}=8 \mathrm{~cm}$. We can easily see that the threshold current is a decreasing function of external cavity reflectivity. This is attributed to the increases of photon lifetime in the EC with $R_{\text {ext }}$. We show also that by increasing 


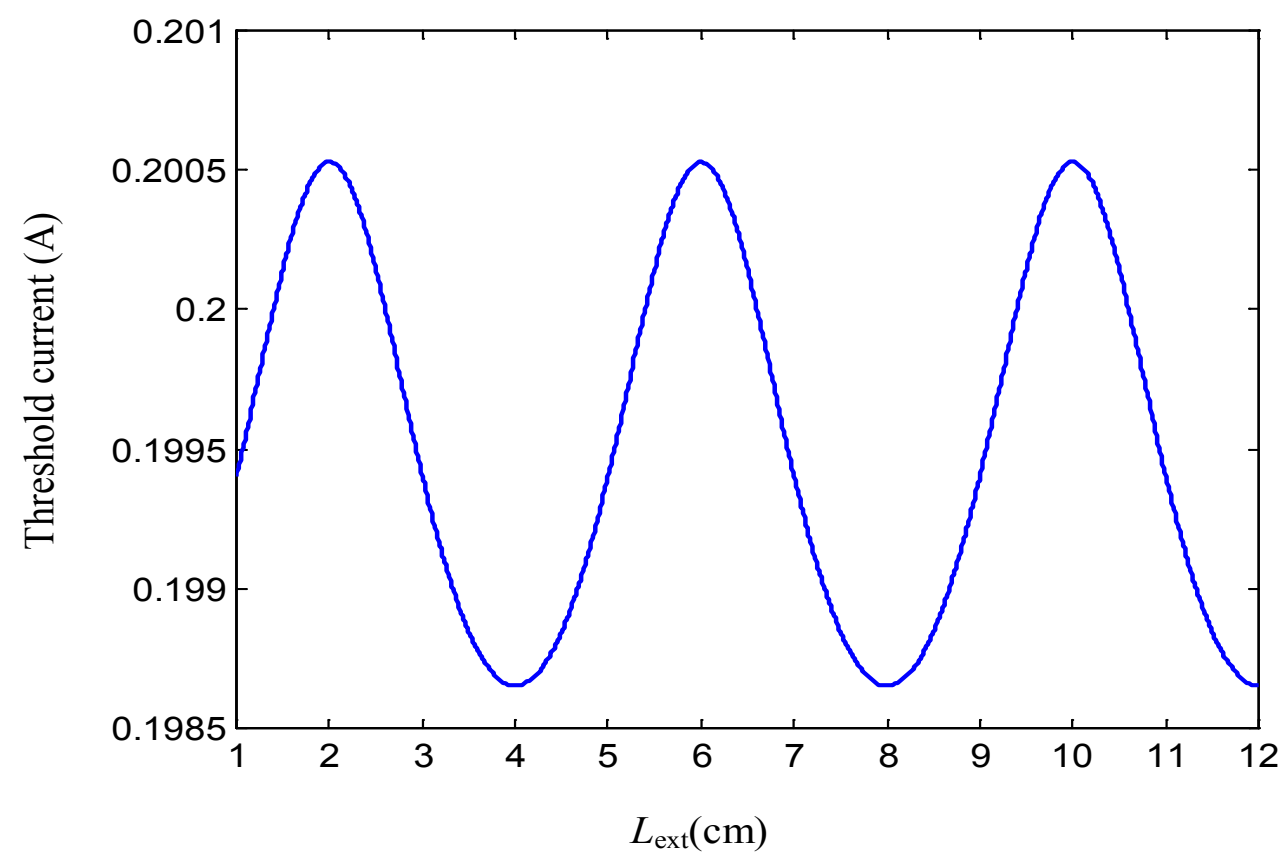

Figure 2: Effect of external cavity length ( $L_{\text {ext }}$ ) on threshold current $I_{t h}^{E C}$. The external cavity reflectivity is $R_{\text {ext }}=90 \%$.

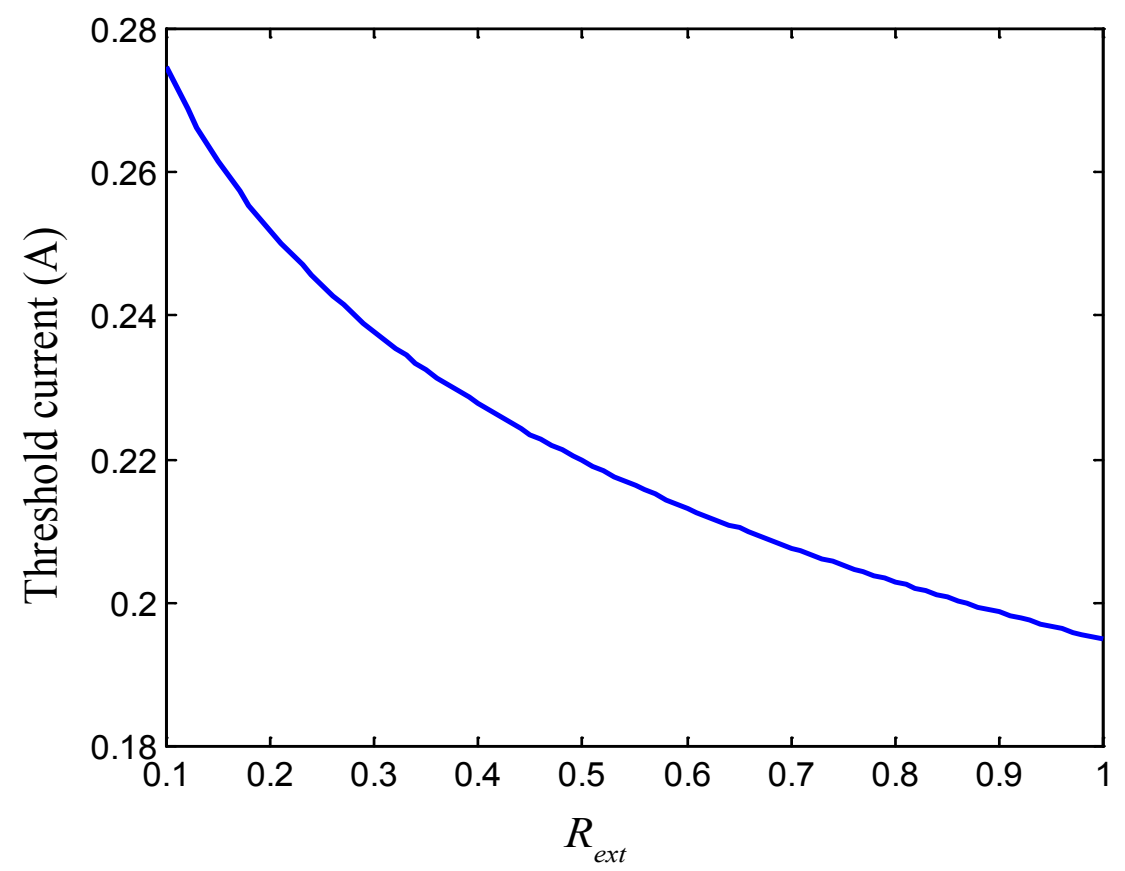

Figure 3: Effect of external cavity reflectivity $\left(R_{\text {ext }}\right)$ on threshold current $I_{t h}^{E C}$. The external cavity length is $L_{\text {ext }}=8 \mathrm{~cm}$.

the external cavity reflectivity from $10 \%$ to $100 \%$, the threshold current decreased from $0.275 \mathrm{~A}$ to $0.195 \mathrm{~A}$.

\section{Dynamical Analysis}

\section{Numerical analysis}

The temporal evolution of the photon numbers $S^{F P}$ and $S^{E C}$ is analyzed by numerically solving the system of nonlinear differential equations 
given in Eq.(1) using the fourth-order Runge-Kutta method. The results are all obtained for an external cavity length $L_{e x t}=8 \mathrm{~cm}$ and an external cavity reflectivity $R_{\text {ext }}=90 \%$. The following initial conditions for electron numbers and photon numbers are: $N_{1}(0)=N_{2}(0)=N_{3}(0)=0$ and $S^{F P}(0)=S^{E C}(0)=0$. In addition, the injection currents are assumed corresponding to the constant value of $I_{t h}^{10 \%}$ (i.e., $I_{i n j}=b I_{t h}^{10 \%}$, where $I_{t h}^{10 \%}=0.275$ A is the threshold current at $R_{e x t}=10 \%$ ).

The temporal evolution of the photon numbers $S^{F P}$ and $S^{E C}$ of the two modes for an injection current $I_{i n j}=2 I_{t h}^{10 \%}$ is displayed in Figure 4. Also shown on the same figure are the three dynamical characteristics times: (i) A delay time interval $\left(t_{d}^{E C}\right)$ where the number of photons in the EC reaches $10 \%$ of its stationary value. In the conditions of Figure 4, this extends from the time origin given by the bias current to about $1.8 \mathrm{~ns}$, (ii) A time taken for $\mathrm{S}^{\mathrm{EC}}$ to rise from $10 \%$ to $90 \%$ of its stationary value. In Figure 4, it extends from $1.8 \mathrm{~ns}$ to about $2.4 \mathrm{~ns}$. This period is called the rise time $\left(\Delta t_{\text {rise }}^{E C}\right)$, (iii) $\mathrm{A}$ long time interval where the laser reaches its stationary regime. In the conditions of Figure 4, this goes at about 3ns after the start of current injection. This period is called the TSSE $\left(t_{s s}^{E C}\right)$. The results of Figure 4 show that the FP number of photons reaches its maximum and stabilizes within less then $0.5 \mathrm{~ns}$, followed by a delay time for the EC number of photons to buildup and to reach its maximum, accompanied by the simultaneous decay of the FP number of photons. This delay time is of around of $1.8 \mathrm{~ns}$.

\section{Derivation of the dynamical characteristics of EC-QC laser}

In this section, we first derive approximate analytical time-dependent solutions of the rate equations above external cavity QC laser threshold and we present our results to derive the expressions that allow for the determination of the dynamical characteristics of external cavity such as $t_{d}^{E C}, \Delta t_{\text {rise }}^{E C}$, and $t_{s s}^{E C}$.

The system of EC-QC laser rate equations are difficult to solve analytically. The derivation of the approximate solutions of the system can be substantiated analytically by employing adiabatic

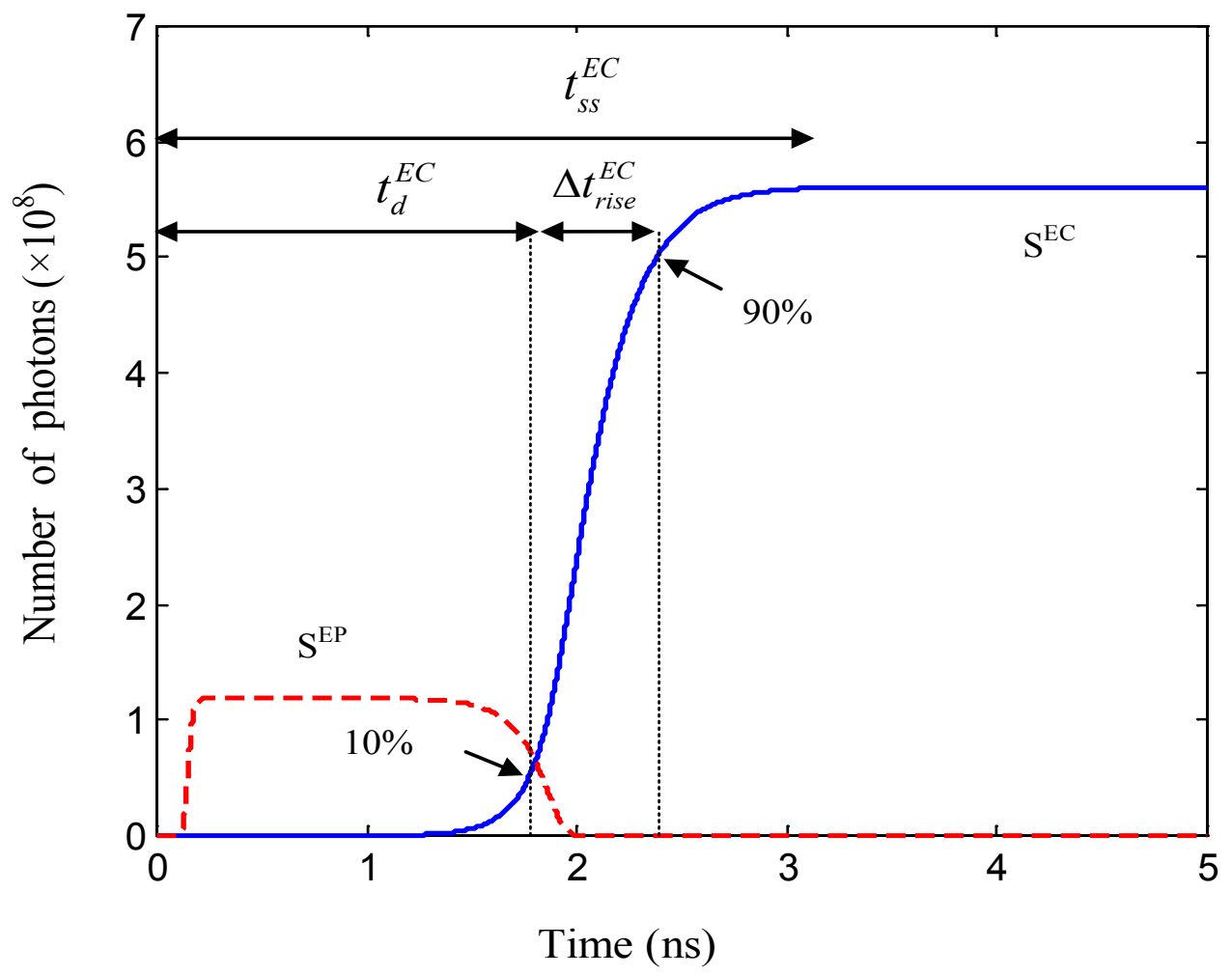

Figure 4: Time evolution of $\mathrm{S}^{\mathrm{FP}}$ and $\mathrm{S}^{\mathrm{EC}}$ for an current injection $I_{i n j}=2 I_{t h}^{10 \%}$. The external cavity length is $L_{e x t}=8 \mathrm{~cm}$ and the external reflectivity $R_{e x t}=90 \%$. We note three distinct times: delay time $\left(t_{d}^{E C}\right)$, rise time $\left(\Delta t_{r i s e}^{E C}\right)$, and TSSE $\left(t_{s s}^{E C}\right)$. 
elimination technique. This analysis is described in [20].

According to the results obtained in [20], we can make the time-dependent of photon numbers $S^{F P}(t)$ and $S^{E C}(t)$ replace $S^{F P}$ and $S^{E C}$ in the Eqs. $(5 a-b)$ and add in these the temporal expressions that describe the overshoot behaviour of number of electrons in the upper and lower laser levels, we obtain for $N_{3}(t)$ and $N_{2}(t)$ the following expressions

$$
\begin{aligned}
& N_{3}(t)=\frac{I_{i n j}}{e} \tau_{3} \frac{\left(1+\frac{\tau_{21}}{\tau_{3}\left(1+\frac{\tau_{21}}{\tau_{31}}\right)}\left(\frac{S^{F P}(t)}{S_{s a t}^{F P}}+\frac{S^{E C}(t)}{S_{s a t}^{E C}}\right)\right.}{1+\frac{S^{F P}(t)}{S_{s a t}^{F P}}+\frac{S^{E C}(t)}{S_{\text {sat }}^{E C}}}\left(1-e^{-\frac{t}{\tau_{3}}}\right), \\
& N_{2}(t)=\frac{I_{i n j}}{e} \tau_{3} \frac{\left(\frac{\tau_{21}}{\tau_{32}}+\frac{\tau_{21}}{\tau_{3}\left(1+\frac{\tau_{21}}{\tau_{31}}\right)}\left(\frac{S^{F P}(t)}{S_{s a t}^{F P}}+\frac{S^{E C}(t)}{S_{s a t}^{E C}}\right)\right)}{1+\frac{S^{F P}(t)}{S_{s a t}^{F P}}+\frac{S^{E C}(t)}{S_{s a t}^{E C}}}\left(1-\frac{e^{-\frac{t}{\tau_{21}}}}{1-\frac{\tau_{3}}{\tau_{21}}}+\frac{e^{-\frac{t}{\tau_{3}}}}{\frac{\tau_{21}}{\tau_{3}}-1}\right) .
\end{aligned}
$$

From Eqs.(12a-b), we deduce the intersubband time-dependent population inversion $\Delta N(t)$ under the effect of external cavity as

$\Delta N(t)=\frac{I_{i j j}}{e}\left(\frac{\xi_{0}+\left(\xi_{1}+\xi_{2}\left(\frac{S^{F P}(t)}{S_{s a t}^{F P}}+\frac{S^{E C}(t)}{S_{s a t}^{E C}}\right)\right) e^{-\frac{t}{\tau_{21}}}-\left(\xi_{3}+\xi_{4}\left(\frac{S^{F P}(t)}{S_{s a t}^{F P}}+\frac{S^{E C}(t)}{S_{s a t}^{E C}}\right)\right) e^{-\frac{t}{\tau_{3}}}}{1+\frac{S^{F P}(t)}{S_{s a t}^{F P}}+\frac{S^{E C}(t)}{S_{s a t}^{E C}}}\right)$,

Where

$$
\begin{aligned}
& \xi_{0}=\tau_{3}\left(1-\frac{\tau_{21}}{\tau_{32}}\right), \\
& \xi_{1}=\frac{\tau_{21}^{2}}{\tau_{32}} \frac{1}{\left(\frac{\tau_{21}}{\tau_{3}}-1\right)}, \\
& \xi_{2}=\frac{\tau_{21}^{2}}{\tau_{3}} \frac{1}{\left(1+\frac{\tau_{21}}{\tau_{31}}\right)\left(\frac{\tau_{21}}{\tau_{3}}-1\right)}, \\
& \xi_{3}=\tau_{3}+\frac{\tau_{3} \tau_{21}}{\tau_{32}} \frac{1}{\left(\frac{\tau_{21}}{\tau_{3}}-1\right)}, \\
& \xi_{4}=\xi_{2}
\end{aligned}
$$

The functions $\exp \left(-t / \tau_{3}\right)$ and $\exp \left(-t / \tau_{21}\right)$ in Eq.(13) vanish quickly and these terms can be omitted. Thus, we rewrite Eq.(13) in the form

$$
\Delta N(t)=\frac{\frac{I_{i n j}}{e} \tau_{3}\left(1-\frac{\tau_{21}}{\tau_{32}}\right)}{1+\frac{S^{F P}(t)}{S_{s a t}^{F P}}+\frac{S^{E C}(t)}{S_{s a t}^{E C}}} .
$$

The expressions for the number of electrons in the upper level $N_{3}(t)$ and the population inversion $\Delta N(t)$ variables are now substituted back into the photon number Eq.(1e) to give the following nonlinear equation, expressed with the help of Eq.(6) as

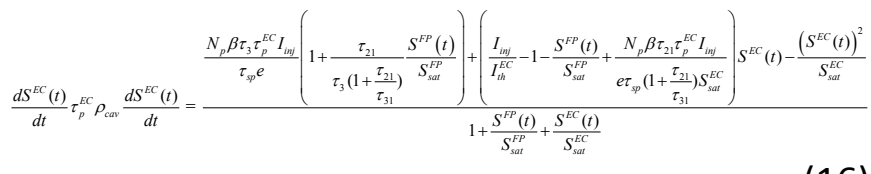

In the interval $I_{t h}^{E C}\left\langle I_{i n j}\left\langle I_{t h}^{F P}\right.\right.$ (i.e., $1\langle b\langle 1.4545$ ), corresponding to a current below the FP threshold, only the EC mode $S^{E C}$ is present and corresponds to a stable state. Then, Eq. (16) reduce to a first order differential equation. By applying the method of partial fraction and integrating for both sides, Eq. (16) becomes

$\int\left(\frac{\alpha}{C S^{E C}(t)+B+\sqrt{B^{2}-4 A C}}+\frac{\beta}{C S^{E C}(t)+B-\sqrt{B^{2}-4 A C}}\right) d S^{E C}(t)=\int \frac{d t}{\tau_{p}^{E C} \rho_{c a v}}$,

Where the coefficients $A, B, C, \alpha$, and $\beta$ are defined as

$$
\begin{aligned}
& A=\frac{N_{p} \beta \tau_{p}^{E C} \tau_{3} I_{i n j}}{\tau_{s p} e}, \\
& B=\frac{1}{2}\left(\frac{I_{i n j}}{\left.I_{t h}^{E C}-1+\frac{N_{p} \beta \tau_{21} \tau_{p}^{E C} I_{i n j}}{\tau_{s p} e\left(1+\frac{\tau_{21}}{\tau_{31}}\right) S_{s a t}^{E C}}\right),}\right. \\
& C=-1 / S_{\text {sat }}^{E C}, \\
& \alpha=\frac{-C}{2}-\frac{C-M B}{2 \sqrt{B^{2}-4 A C}}, \\
& \beta=\frac{-C}{2}+\frac{C-M B}{2 \sqrt{B^{2}-4 A C}},
\end{aligned}
$$

Now, we derive the times $t_{d}^{E C}, \Delta t_{\text {rise }}^{E C}$, and $t_{s s}^{E C}$ using Eq.(17).

Derivation of the delay time: The delay time $t_{d}^{E C}$ is the time elapses between the moment the bias is applied and the time the EC photon number reaches $10 \%$ of its stationary value. To derive $t_{d}^{E C}$ we apply Eq.(17) and integrating it from 0 to $10 \%$ 
value $S^{E C}$ for number of photons, and from zero to $t_{d}^{E C}$ for time. i.e.,

$\int_{0}^{0.1 S^{E C}}\left(\frac{\alpha}{C S^{E C}(t)+B+\sqrt{B^{2}-4 A C}}+\frac{\beta}{C S^{E C}(t)+B-\sqrt{B^{2}-4 A C}}\right) d S^{E C}(t)=\int_{0}^{t_{d}^{E C}} \frac{d t}{\rho_{c a v} \tau_{p}^{E C}}$.

The integration gives us

$t_{d}^{E C}=\frac{\rho_{c a v} \tau_{p}^{E C}}{\frac{I_{i n j}}{I_{t h}^{E C}}-1}\left(\ln \left(1+\frac{1}{10} \frac{\tau_{s p} \eta_{r}}{\beta \tau_{3}} \frac{I_{t h}^{E C}}{I_{i n j}}\left(\frac{I_{i n j}}{I_{t h}^{E C}}-1\right)^{2}\right)-\frac{I_{i n j}}{I_{t h}^{E C}} \ln \left(\frac{9}{10}\right)\right)$.

Derivation of the rise time: Now, to compute the rise time $\Delta t_{\text {rise }}^{E C}$, i.e., the time taken for $S^{E C}$ to rise from $10 \%$ to $90 \%$ of its stationary value, we use the Eq.(17) and integrating it from $10 \%$ to $90 \%$ value $S^{E C}$ for number of photons, and from zero to $\Delta t_{\text {rise }}^{E C}$ for time. i.e.,

$\int_{0.1 S^{E C}}^{0.9 S^{E C}}\left(\frac{\alpha}{C S^{E C}(t)+B+\sqrt{B^{2}-4 A C}}+\frac{\beta}{C S^{E C}(t)+B-\sqrt{B^{2}-4 A C}}\right) d S^{E C}(t)=\int_{0}^{\Delta t_{\text {ree }}^{E C}} \frac{d t}{\rho_{c a v} \tau_{p}^{E C}}$.
The integration gives us after some algebra

$$
\Delta t_{r i s e}^{E C}=\left(\frac{I_{i n j} / I_{t h}^{E C}+1}{I_{i n j} / I_{t h}^{E C}-1}\right) \rho_{c a v} \tau_{p}^{E C} \ln 9 .
$$

Derivation of the TSSE: The TSSE $\left(t_{s s}^{E C}\right)$, i.e., the time that elapses between the moment the bias is applied and the time the EC photon number reaches its stationary value, we use the Eq.(17) and integrating it from zero to steady-state value $S^{E C}$ for number of photons, and from zero to $t_{s s}^{E C}$ for time. i.e.,

$$
\int_{0}^{S^{E C}}\left(\frac{\alpha}{C S^{E C}(t)+B+\sqrt{B^{2}-4 A C}}+\frac{\beta}{C S^{E C}(t)+B-\sqrt{B^{2}-4 A C}}\right) d S^{E C}(t)=\int_{0}^{t c C} \frac{d t}{\rho_{c u v} \tau_{p}^{E C}} \text {. }
$$

Finally, the steady-state under the effect of external cavity occurs approximately at the time

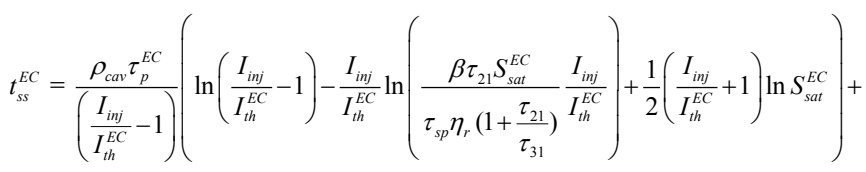
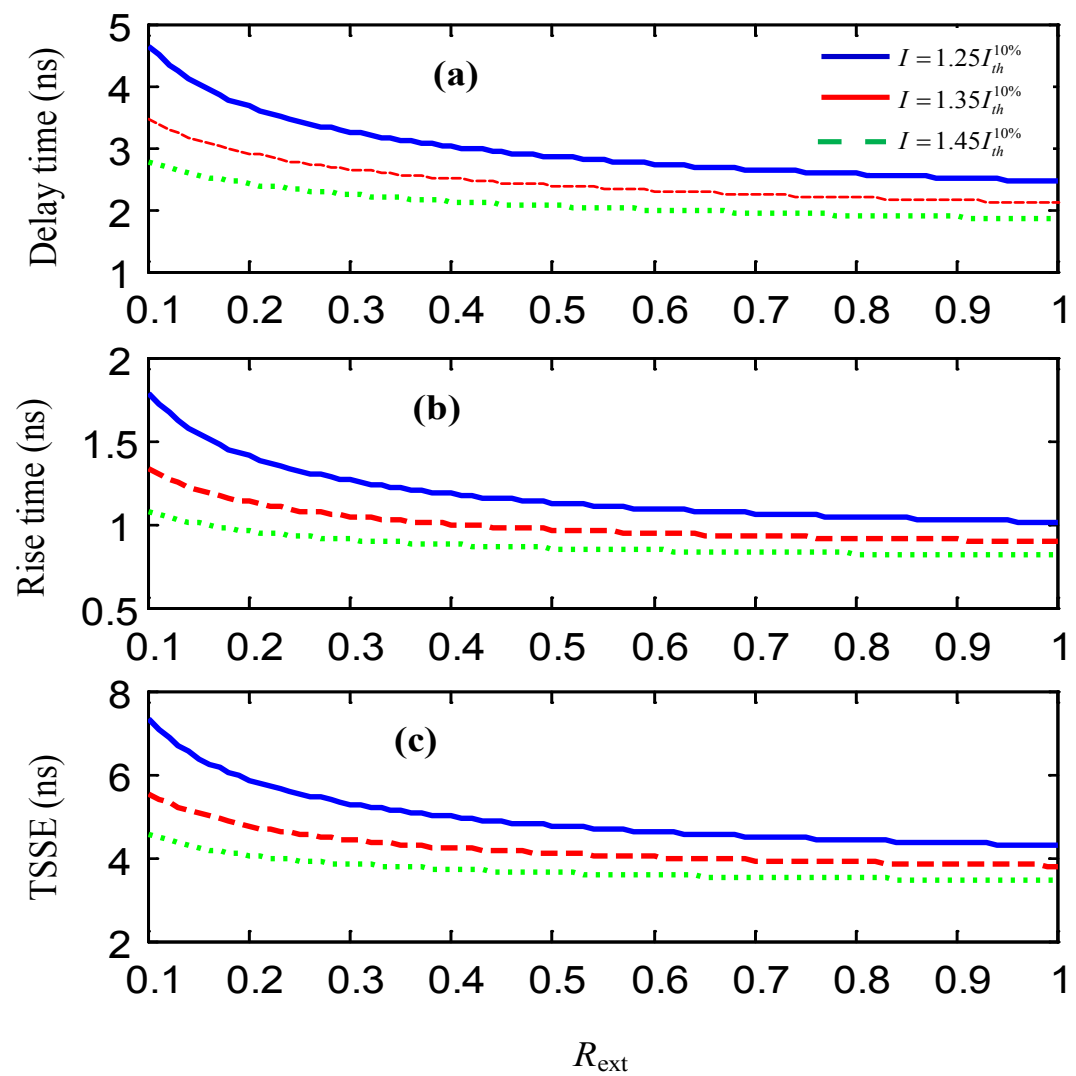

Figure 5: Effect of external cavity reflectivity $\left(R_{\text {ext }}\right)$ on delay time $t_{d}^{E C}$, rise time $\Delta t_{\text {rise }}^{E C}$ and TSSE $t_{s s}^{E C}$ for three current injection. Blue solid line $\left(I_{i n j}=1.25 I_{t h}^{10 \%}\right)$, red dashed line $\left(I_{i n j}=1.35 I_{t h}^{10 \%}\right)$, and green dotted one ( $I_{i n j}=1.45 I_{t h}^{10 \%}$ ). The external cavity length is $L_{e x t}=8 \mathrm{~cm}$. 


$$
+\rho_{c a v} \tau_{p}^{E C}\left(1+\frac{\tau_{21}}{\tau_{31}}\right) \frac{\eta_{r} \tau_{s p}}{\beta \tau_{21}} \ln \left(\frac{\beta \tau_{21}^{2}}{\tau_{s p} \tau_{3} \eta_{r}\left(1+\frac{\tau_{21}}{\tau_{31}}\right)^{2}}+1\right)
$$

We shows in Figure $5 a$, Figure $5 b$ and Figure $5 c$ the variation of the (a) delay time $t_{d}^{E C},(\mathrm{~b})$ rise time $\Delta t_{r i s e}^{E C}$, and (c) TSSE $t_{s s}^{E C}$ of the EC-QC laser as a function of the external cavity reflectivity which we vary from $10 \%$ to $100 \%$. The results are all obtained for three values of the injection current below external cavity threshold. It is worthwhile to stress the strong decrease of $t_{d}^{E C}, \Delta t_{\text {rise }}^{E C}$, and $t_{s s}^{E C}$ as the external reflectivity $R_{\text {ext }}$ increases from its minimal value upward. This decrease are due to the strong dependence of the threshold current $I_{t h}^{E C}$ on photon lifetime $\tau_{p}^{E C}$, where $\tau_{p}^{E C}$ is strongly affected by the external reflectivity value. We also see that the times $t_{d}^{E C}, \Delta t_{\text {rise }}^{E C}$, and $t_{s s}^{E C}$ decrease with increasing current injection. The physical reason of this result is apparent: High external cavity reflectivity leads to low external cavity threshold values, thereby increasing the ratio of bias to threshold current. Finally, the different times are of the order of few nanoseconds.

We conclude our analysis with Figure 6a, Figure
$6 b$, and Figure $6 c$, where we show the variation of the delay time $t_{d}^{E C}$, (b) rise time $\Delta t_{\text {rise }}^{E C}$, and (c) TSSE $t_{s s}^{E C}$ of the EC-QC laser as a function of the external cavity length $L_{e x t}$ which we vary from 1 to 12 $\mathrm{cm}$. The results are all obtained for three values of the injection current below EC threshold. We can immediately see that the times $t_{d}^{E C}, \Delta t_{\text {rise }}^{E C}$, and $t_{s s}^{E C}$ , starting respectively from $0.5 \mathrm{~ns}, 0.25 \mathrm{~ns}$, and 1 ns at $L_{\text {ext }}=1 \mathrm{~cm}$, increase with the external cavity length and depend strongly on current injection at high $L_{e x t}$. The times $t_{d}^{E C}$ and $\Delta t_{\text {rise }}^{E C}$, increase significantly when the current injection is reduced, and these lead to an important increases of the TSSE $t_{s s}^{E C}$.

\section{Conclusion}

Using a simple rate equations model, we developed an analytical scheme to derive the dynamical characteristics such as delay time, rise time, and TSSE as functions of current injection and the external cavity parameters of the system of a QC laser. We find as excepted that the threshold current decreases from $0.275 \mathrm{~A}$ at $R_{\text {ext }}=10 \%$ to $0.195 \mathrm{~A}$ for $R_{\text {ext }}=100 \%$. Our numerical results also show that the current injection and the external cavity parameters affect the dynamical characteristics quite strongly.
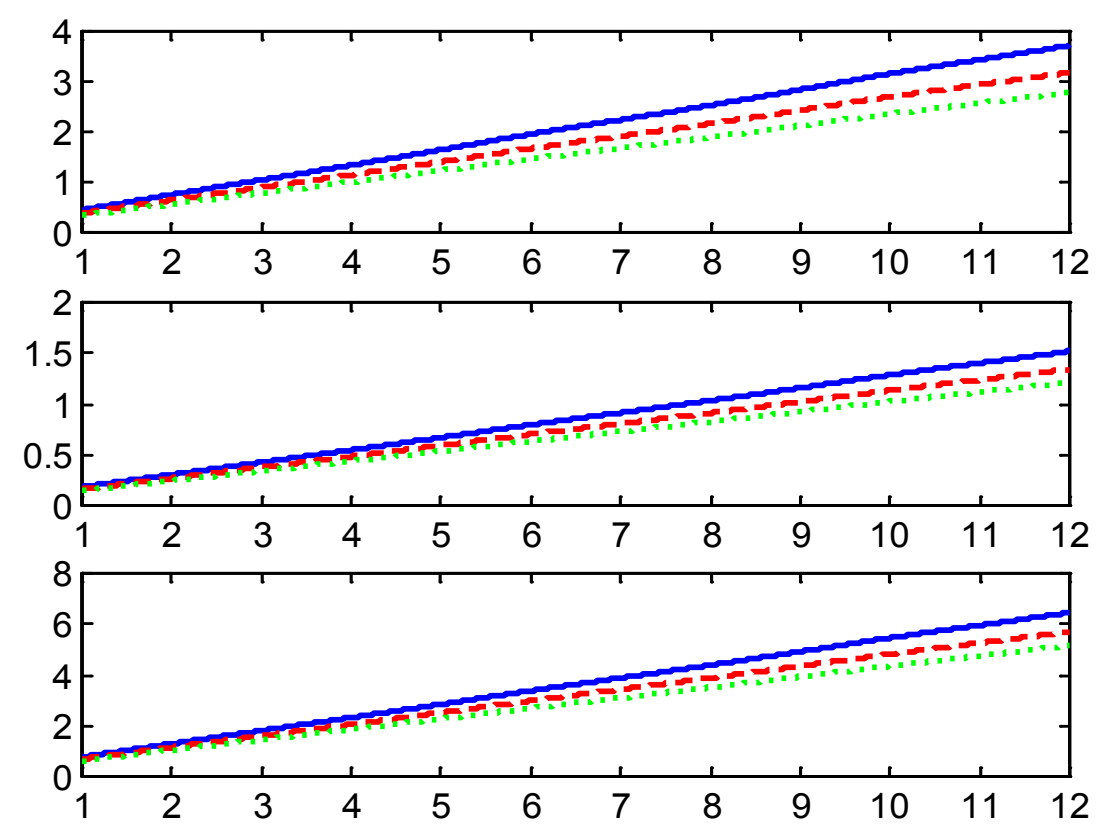

Figure 6: Effect of external cavity length $\left(L_{\text {ext }}\right)$ on delay time $t_{d}^{E C}$, rise time $\Delta t_{\text {rise }}^{E C}$ and TSSE $t_{s s}^{E C}$ for three current injection. Blue solid line $\left(I_{i n j}=1.25 I_{t h}^{10 \%}\right)$, red dashed line $\left(I_{i n j}=1.35 I_{t h}^{10 \%}\right)$, and green dotted one ( $\left.I_{i n j}=1.45 I_{t h}^{10 \%}\right)$. The external cavity reflectivity is $R_{\text {ext }}=90 \%$. 


\section{Acknowledgments}

The author is grateful to the Algerian Ministry of Higher Education for a research grant.

\section{References}

1. Faist J, Capasso F, Sivco DL, Sirtori C, Hutchinson AL, Cho AY (1994) Quantum cascade laser. Science 264: 553-556.

2. Luo GP, Peng C, Le HQ, Pei SS, Hwang WY, et al. (2001) Grating-tuned external-cavity quantum-cascade semiconductor lasers. Appl Phys Lett 78: 28342836.

3. Luo GP, Peng C, Le HQ, Pei SS, Lee $H$, et al. (2002) Broadly wavelength-tunable external cavity, mid-infrared quantum cascade lasers. IEEE J Quantum Electron 38: 486-494.

4. Totschnig G, Winter F, Pustogov V, Faist J, Muller A (2002) Mid-infrared external cavity quantum-cascade laser. Opt Lett 27: 1788-1790.

5. Peng C, Luo GP, Le HQ (2003) Broadband, continuous, and fine-tune properties of external-cavity thermoelectric-stabilized mid-infrared quantum-cascade lasers. App Opt 42: 4877-4882.

6. Peng C, Zhang HL, Le HQ (2003) Mid-infrared external-cavity two-segment quantum cascade laser. App Phys Lett 83: 4098-4100.

7. Maulini R, Beck M, Faist J, Gini E (2004) Broadband tuning of external cavity bound-to-continuum quantum-cascade lasers. Appl Phys Lett 84: 1659-1661.

8. Maulini R, Yarekha DA, Bulliard JM, Giovannini M, Faist J (2005) Continuous wave operation of a broadly tunable thermoelectrically cooled external cavity quantum cascade laser. Opt Lett 30: 2584-2586.

9. Maulini R, Mohan A, Giovannini M, Faist J, Gini E (2006) External cavity quantum-cascade laser tunable from 8.2 to $10.4 \mu \mathrm{m}$ using a gain element with a heterogeneous cascade. Appl Phys Lett 88: 201113.
10. Wysocki G, Lewicki R, Curl RF, Tittel FK, Diehl L, et al. (2008) Widely tunable mode-hop free external cavity quantum cascade lasers for high resolution spectroscopy and chemical sensing. Appl Phys B 92: 305-311.

11.Maulini R, Dunayevskiy I, Lyakh A, Tsekoun A, Patel $C$, et al. (2009) Widely tunable high-power external cavity quantum cascade laser operating in continuous-wave at room temperature. Electron Lett 45: 107-108.

12. Hinkov B, Yang Q, Fuchs F, Bronner W, Kohler K, et al. (2009) Time-resolved characterization of external-cavity quantum-cascade lasers. App Phys Lett 94: 221105.

13. Hugi A, Maulini R, Faist J (2010) External cavity quantum cascade laser. Semicond Sci Technol 25: 083001.

14. Yang QK, Hinkov B, Fuchs F, Bronner W, Köhler K, et al. (2010) Rate equations analysis of external-cavity quantum cascade lasers. J Appl Phys 107: 043109.

15. Hamadou A (2018) The effect of external cavity reflectivity on the dynamic behaviors of quantum cascade laser. Chines J Phys 56: 1286-1295.

16. Iotti RC, Rossi F (2005) Microscopic theory of semiconductor-based optoelectronic devices. Rep Prog Phys 68: 2533-2571.

17.Yamanishi M, Edamura T, Fujita K, Akikusa N, Kan H (2008) Theory of the intrinsic line width of quantum cascade lasers: hidden reason for the narrow linewidth and line-broadening by thermal photons. IEEE J Quantum Electron 44: 12-29.

18. Maulini R (2009) Broadly tunable mid-infrared quantum cascade lasers for spectroscopic applications. VDM Verlag, Saarbrücken, Germany.

19.Olsson A, Tang CL (1981) Coherent optical interference effects in external-cavity semiconductor lasers. IEEE J Quantum Electron 17: 1320-1323.

20. Hamadou A (2015) Analytical investigation of the dynamics behaviors of quantum cascade laser. Optics commun 335: 271-278. 